\title{
ANÁLISES PARAMÉTRICAS E NÃO-PARAMÉTRICAS PARA DETERMINAÇÃO DA ADAPTABILIDADE E ESTABILIDADE DE GENÓTIPOS DE SOJA ${ }^{1}$
}

\section{PARAMETRIC AND NON-PARAMETRIC ANALYSES TO THE DETERMINATION OF STABILITY AND ADAPTABILITY OF SOYBEAN GENOTYPES}

\author{
Hélio Bandeira BARROS 2 \\ Tuneo SEDIYAMA ${ }^{3}$ \\ Rita de Cássia TEIXEIRA 4 \\ Cosme Damião $\mathrm{CRUZ}^{5}$
}

\begin{abstract}
RESUMO
Este trabalho teve o objetivo de avaliar a estabilidade e a adaptabilidade produtiva de 30 genótipos de soja [Glycine max (L.) Merrill], em seis ambientes no Estado do Mato Grosso. O delineamento experimental foi em blocos ao acaso, com quatro repetições. Os ensaios foram conduzidos no ano de 2004/05 nos municípios de Rondonópolis, Campo Verde, Nova Brasilândia e Vera. Para avaliação da adaptabilidade e estabilidade, utilizaram-se os métodos de Eberhart e Russell (1966), Lin e Binns (1988) modificado por Carneiro (1998), Annicchiarico (1992) e Centróide (Rocha et al., 2005). A produtividade média de grãos variou de $2.837 \mathrm{~kg} \mathrm{ha}^{-1}$ (Rondonópolis III) a $3.701 \mathrm{~kg} \mathrm{ha}^{-1}$ (Vera), com média geral entre os ambientes de 3.200 $\mathrm{kg} \mathrm{ha}^{-1}$. Pela metodologia proposta por Eberhart e Russell, as linhagens SL 2238. SL 1949 SL 31, SL 2382 e os padrões Uirapuru e M-Soy 8411 apresentaram as maiores médias, ampla adaptabilidade $\left(\beta_{1}=1\right)$ e $\sigma_{d i}^{2}$ significativos, ou seja, baixa estabilidade. Pelas metodologias de Lin e Binns, Annicchiarico e Centróide, as linhagens SL 2238, SL 712, SL 1949 e a cultivar Uirapuru foram classificadas como as mais produtivas, adaptadas e estáveis, havendo, portanto, coerência entre tais metodologias. Com base nas metodologias de Lin e Binns (1988), Annicchiarico (1992) e Centróide (Rocha et al. 2005), as linhagens SL 2238, SL 1949 e SL 712 apresentaram superioridade em termos de adaptabilidade, estabilidade e desempenho para a produtividade de grãos.
\end{abstract}

Palavras-chave: Glycine max, produtividade de grãos, genótipo x ambiente.

\begin{abstract}
This work aimed to evaluate the yield stability and adaptability of 30 soybean genotypes [Glycine max (L.) Merr.] in six environments at the State of Mato Grosso, Brazil. A randomized complete blocks design with four replicates was used. The trials were carried out at 2004/05 experimental years at the counties of Rondonópolis, Campo Verde, Nova Brasilândia and Vera. To evaluate the adaptability and stability, the methods developed by Eberhart and Russell (1966), Lin and Binns (1988), and modified by Carneiro (1998), Annicchiarico (1992) and Centroid Method (Rocha et al., 2005) were used. The grain yield mean varied from $2837 \mathrm{~kg} \mathrm{ha}^{-1}$ (Rondonópolis III) to $3701 \mathrm{~kg} \mathrm{ha}^{-1}$ (Vera) with general mean among the environments of $3200 \mathrm{~kg}$ $\mathrm{ha}^{-1}$. According to the methodologies developed by Eberhart and Russel, the SL 2238, SL 1949, SL 31, SL 2382 lines and the standards Uirapuru and M-Soy 8411 , presented the highest means, wide adaptability $\left(\beta_{1}=1\right)$ and $\sigma_{o j}^{2}$ statistically significant, which means a low stability of such materials. The SL 2238, SL 712, SL 1949 lines and the Uirapuru cultivar were classified by having the highest grain yield, adaptability and stability, according to the methodology proposed by Lin and Binns, Annicchiarico and Centroid, having, therefore, consistency among such methodologies. The SL 2238, SL 1949 and SL 712 lines presents superiority in terms of adaptability, stability and performance for grain yield, according to Lin and Binns (1988), Annicchiarico (1992) and Centroid (Rocha et al., 2005) methodologies.
\end{abstract}

Key-words: Glycine max, grain yield, genotype x environment.

1. Trabalho extraído da Tese de Doutorado do primeiro autor, pela Universidade Federal de Viçosa, Departamento de Fitotecnia.

2. Engenheiro Agrônomo, Doutor em Fitotecnia, Professor adjunto pela Universidade Federal do Tocantins, Campus de Gurupi (TO). E-mail: bhb2006@yahoo.com.br. Autor para correspondência.

3. Engenheiro Agrônomo, PhD em Genética e Melhoramento de Plantas, Professor do Departamento de Fitotecnia da UFV.

4. Economista Doméstica, Mestre, Pesquisadora do Campo experimental Bacuri.

5. Engenheiro Agrônomo, Doutor em Agronomia, Professor do Departamento de Biologia Geral da UFV 


\section{INTRODUÇÃO}

Para a maioria das características de importância econômica, em especial a produtividade de grãos, o fenótipo de soja a ser selecionado depende do genótipo, do ambiente e da interação do genótipo $x$ ambiente. Essa interação ocorre devido à inconsistência do desempenho dos genótipos nos vários ambientes, refletindo nas diferentes respostas dos genótipos às mudanças ambientais. Considerando as inúmeras variações ambientais em que a soja é comumente submetida no Brasil, é esperado que a interação genótipo $x$ ambiente assuma papel fundamental na manifestação fenotípica, devendo, portanto, ser estimada e considerada no programa de melhoramento genético e na indicação de cultivares (PRADO et al., 2001).

Quando o comportamento de duas cultivares são concordantes em dois ambientes distintos, a interação é chamada de interação simples, não acarretando maiores problemas. Entretanto, quando as cultivares possuem comportamento diverso, a interação é denominada complexa. Considerando um número maior de ambientes e de cultivares, a presença de interação complexa quase sempre indica a existência de cultivares especificamente adaptadas a ambientes particulares, bem como de outras com adaptação mais ampla, porém sem alto potencial produtivo (RAMALHO et al., 1993).

Em um programa de melhoramento, a avaliação de genótipos visando a identificação e recomendação de materiais superiores em diferentes ambientes é considerada por muitos autores como uma das etapas mais importante, trabalhosa e onerosa (SILVA e DUARTE, 2006; MAIA et al., 2006; ROCHA et al., 2005; NUNES et al., 2002; ATROCH et al., 2000; PRADO et al., 2001 e FARIAS, et al., 1997). Isso porque exige a condução de experimentos precisos e em uma grande amplitude de condições ambientais. Existem disponíveis, na literatura, vários métodos para estudo e quantificação da interação genótipo x ambiente entre eles: o método tradicional; PLAISTED e PETERSON, (1959); FINLAY e WILKINSON, (1963); WRICKE (1965); EBERHART e RUSSELL (1966); PERKINS e JINKS (1968); FREEMAN e PERKINS (1971); TAI (1971); SILVA e BARRETO (1986); LIN e BINNS (1988); CRUZ et al. (1989) e ANNICCHIARICO (1992). A diferença entre os métodos origina-se nos próprios conceitos de estabilidade e nos procedimentos biométricos empregados para medi-la.

Os métodos baseados em regressão relacionam as respostas individuais dos genótipos com o efeito do ambiente, que geralmente é estimado utilizando o índice ambiental associado tanto à regressão linear simples quanto à regressão linear bissegmentada (CRUZ e REGAZZI, 1997). Como exigências desses métodos, destacam-se: o número mínimo de ambientes para análise (três para os métodos que utilizam regressão linear simples e seis para os que utilizam regressão linear bissegmentada), o maior número de parâmetros que devem ser simultaneamente avaliados para a recomendação, e um problema de ordem estatística, a existência de dependência entre o índice ambiental utilizado para classificar os ambientes e a produtividade média da cultivar (CRUZ et al., 1989).

Segundo EBERHART e RUSSELL (1966), a cultivar ideal é aquela que tem alta produtividade, coeficiente de regressão igual à unidade e desvio de regressão não significativo. LIN et al. (1986) apresentaram críticas aos métodos que se baseiam no desvio da regressão como parâmetro de estabilidade. Segundo os autores, esse parâmetro serve apenas para indicar o ajuste dos dados à equação obtida, ao invés de maior ou menor estabilidade da cultivar. Reiteram que, baixa adaptação representada por $\sigma_{d i}^{2}$ elevado ou coeficiente de determinação $\left(F_{i}^{2}\right)$ pequeno, deve ser interpretado como indicativo de que o uso do modelo de regressão para estimar a estabilidade não é apropriado e que alternativas devam ser investigadas. Entretanto, a facilidade na interpretação dos resultados popularizaram seu emprego.

Metodologias baseadas em análise de componentes principais, embora rotineiramente utilizadas em programas de melhoramento em estudos de diversidade genética, são pouco utilizadas em estudos da interação genótipo $x$ ambiente (ROCHA et al., 2005). Nesse trabalho, a metodologia baseada na análise de componentes principais denominada de Centróide, foi utilizada para representar a variação da performance dos genótipos nos ambientes em uma dispersão no plano com poucos eixos, o que permite uma análise simultânea do desempenho de um número elevado de genótipos em virtude da facilidade de interpretação dos resultados.

O objetivo deste trabalho foi avaliar, com base na produtividade de grãos, na estabilidade e na adaptabilidade, 24 genótipos de soja de ciclo precoce e seis cultivares, em seis ambientes do Estado do Mato Grosso, através dos métodos de EBERHART e RUSSELL (1966); LIN e BINNS (1988), modificado por CARNEIRO (1998); ANNICCHIARICO (1992) e Centróide (ROCHA et al. 2005).

\section{MATERIAL E MÉTODOS}

Foram utilizados os dados de produtividade de grãos $\left(\mathrm{kg} \mathrm{ha}^{-1}\right)$ dos ensaios finais de competição de genótipos de soja de ciclo precoce do Programa de Melhoramento Genético do Campo Experimental Bacuri e Sales Agropecuária, conduzidos em Mato Grosso, no ano agrícola 2004/05, nas localidades de Nova Brasilândia, Campo Verde, Vera e Rondonópolis (Tabela 1). Em Rondonópolis foram conduzidos três ensaios, denominados Rondonópolis I, Rondonópolis II e Rondonópolis III, que corresponderam a diferentes épocas de semeadura. Foram avaliados 30 genótipos, entre eles, seis cultivares testemunhas (Conquista, Emgopa 315, M-Soy 8329, M-Soy 8411, Uirapuru e Xingu). 
BARROS, H.B. et al. Análises paramétricas e não-paramétricas...

TABELA 1 - Altitude, latitude, longitude e data de semeadura de ensaios de competição de linhagens de soja, de ciclo precoce, no Estado do Mato Grosso, na safra 2004/2005

\begin{tabular}{lcccc}
\hline \multicolumn{1}{c}{ Ambiente } & Altitude $(\mathrm{m})$ & Latitude & Longitude & Data de semeadura \\
\hline Rondonópolis I & 227 & $16^{\circ} 28^{\prime} 15^{\prime \prime} \mathrm{S}$ & $54^{\circ} 38^{\prime} 08^{\prime \prime} \mathrm{W}$ & $07 / 11 / 2004$ \\
Rondonópolis II & 227 & $16^{\circ} 28^{\prime} 15^{\prime \prime} \mathrm{S}$ & $54^{\circ} 38^{\prime} 08^{\prime \prime} \mathrm{W}$ & $29 / 11 / 2004$ \\
Rondonópolis III & 227 & $16^{\circ} 28^{\prime} 15^{\prime \prime} \mathrm{S}$ & $54^{\circ} 38^{\prime} 08^{\prime \prime} \mathrm{W}$ & $19 / 12 / 2004$ \\
Nova Brasilândia & 540 & $14^{\circ} 57^{\prime} 25^{\prime \prime} \mathrm{S}$ & $54^{\circ} 57^{\prime} 56^{\prime \prime} \mathrm{W}$ & $04 / 12 / 2004$ \\
Campo Verde & 736 & $15^{\circ} 32^{\prime} 48^{\prime \prime} \mathrm{S}$ & $55^{\circ} 10^{\prime} 08^{\prime \prime} \mathrm{W}$ & $14 / 12 / 2004$ \\
Vera & 383 & $12^{\circ} 18^{\prime} 21^{\prime \prime} \mathrm{S}$ & $55^{\circ} 19^{\prime} 01^{\prime \prime} \mathrm{W}$ & $15 / 11 / 2004$ \\
\hline
\end{tabular}

Os experimentos foram instalados no delineamento de blocos completos casualizados, com quatro repetições. As parcelas foram formadas por quatro fileiras de plantas $(5 \mathrm{~m})$, espaçadas em $0,45 \mathrm{~m}$ entre as fileiras. A área útil da parcela foi de $3,6 \mathrm{~m}^{2}$, tendo sido colhidas as duas fileiras centrais, desprezando-se $0,5 \mathrm{~m}$ de bordadura nas extremidades.

Foram realizadas análises de variância individuais (Tabela 2), seguindo-se uma análise de variância conjunta. A fim de implementar tais análises, utilizou-se o aplicativo computacional em genética e estatística - GENES (CRUZ, 2001). Na análise conjunta, avaliou-se primeiramente a homogeneidade das variâncias residuais dos experimentos (QMR), verificada pela razão entre o maior e menor quadrado médio residual dos ensaios $(1,7493)$. Segundo PIMENTEL-GOMES (1990) as variâncias são consideradas homogêneas quando a relação entre o maior e o menor QMR é menor que 7,0. A decomposição do quadrado médio da interação foi estimada de acordo a metodologia proposta por CRUZ e CASTOLDI (1991) em que a parte complexa é expressa por:

$$
C=\sqrt{(1-r)^{3} Q_{1} Q_{2}}
$$

em que: $Q_{1}=$ maior quadrado médio entre genótipos nos dois locais; $Q_{2}=$ menor quadrado médio entre genótipos nos dois locais; $r=$ coeficiente de correlação simples entre genótipos nos dois locais.

TABELA 2 - Produtividade média de grãos $\left(\bar{Y}_{j}\right)$, variância residual (QMR) e coeficiente de variação (CV) de ensaios de competição de linhagens de soja, de ciclo precoce, no Estado do Mato Grosso, na safra $2004 / 2005$

\begin{tabular}{lccc}
\hline \multicolumn{1}{c}{ Ambiente } & $\bar{Y}_{. j}$ & QMR & CV (\%) \\
\hline Rondonópolis I & 3109,1 & 110909,0129 & 10,71 \\
Rondonópolis II & 3177,3 & 135669,5842 & 11,59 \\
Rondonópolis III & 2836,5 & 194012,0908 & 15,52 \\
Nova Brasilândia & 3145,3 & 159378,2478 & 12,69 \\
Campo Verde & 3234,2 & 131955,3946 & 11,23 \\
Vera & 3700,5 & 149413,6589 & 10,44 \\
\hline
\end{tabular}

A análise de adaptabilidade e estabilidade fenotípica dos genótipos foi feita pelos seguintes métodos: EBERHART e RUSSELL (1966), LIN e BINNS (1988) modificado por CARNEIRO (1998), ANNICCHIARICO (1992) e Centróide (ROCHA et al. 2005).

A metodologia e EBERHART e RUSSELL (1966) usa, na avaliação individual dos genótipos, a produtividade média do genótipo $\left(\mu_{i}\right)$, o seu coeficiente de regressão $\left(\beta_{1}\right)$ e a variância dos desvios dessa regressão $\left(\sigma_{\alpha^{j}}^{2}\right)$. Seus respectivos estimadores são dados por:

$$
\begin{aligned}
& \overline{Y_{i}}=\frac{\sum_{j} Y_{i j}}{a} \\
& \hat{\beta}_{i}=\frac{\sum_{j} Y_{i j} I_{j}}{\sum_{j} I_{j}^{2}} \\
& \text { em que } \\
& I_{j}=\frac{\sum_{j} Y_{i j}}{g}-\frac{\sum_{i} \sum_{j} Y_{i j}}{a g} \quad \text { (índice ambiental) }
\end{aligned}
$$

$$
\hat{\mathcal{\sigma}}_{d i}^{2}=\frac{\left[\sum_{j} Y_{i j}^{2}-\left(\sum_{j} Y_{i j}\right)^{2} / a\right]-\left(\sum_{j} Y_{i j} I_{j}\right)^{2} / \sum_{j} I_{j}^{2}}{a-2}
$$

A estatística de estabilidade e adaptabilidade $P_{i}$, adotada pelo método de LIN e BINNS (1988), é obtida por:

$$
P_{i}=\frac{\sum_{j=1}^{a}\left(Y_{i j}-M_{j}\right)^{2}}{2 a}
$$

em que $M_{j}$ é a produtividade máxima entre todos os genótipos, no j-ésimo ambiente. O genótipo estável é aquele que apresentar o menor índice $P_{i}$. 
A estimativa da MAEC (medida de adaptabilidade e estabilidade de comportamento) fornece direcionamento da resposta aos diferentes tipos de ambientes. Assim, a recomendação geral é feita com base no $P_{\text {i }}$ original de LIN e BINNS (1988), e para ambientes favoráveis e desfavoráveis, conforme a decomposição proposta por CARNEIRO (1998), estimada por:

$$
P_{i f}=\frac{\sum_{j=1}^{f}\left(Y_{i j}-M_{j}\right)^{2}}{2 f}
$$

em que: $f$ = número de ambientes favoráveis.

Da mesma forma para ambientes desfavoráveis, cujos índices são negativos:

$$
P_{i d}=\frac{\sum_{j=1}^{d}\left(Y_{i j}-M_{j}\right)^{2}}{2 d}
$$

onde: $\mathrm{d}$ = número de ambientes desfavorá-

veis.

O método de ANNICCHIARICO (1992) baseia-se no chamado índice de confiança genotípico, estimado por:

$$
W_{i(g)}=A_{i(g)}-z_{(1-\alpha)} \mathcal{O}_{Z_{I(k)}}
$$

considerando-se todos os ambientes, em que $\hat{H_{i}}(\mathrm{~g})$ é a média porcentual dos genótipos i; $\hat{\mathcal{O}}_{Z_{I(\mathrm{p})}}$ é o desvio padrão $Z_{(1-\omega)}$ valores de $Z_{\mathrm{ij}}$, associado ao i-ésimo genótipo; é o percentil da função de distribuição normal padrão. $O$ índice de confiança adotado foi de $75 \%$, ou seja, $\alpha=0,25$.

$\mathrm{O}$ método centróide, segundo ROCHA et al. (2005), baseia-se na comparação de valores de distância cartesiana entre os genótipos e quatro referências ideais (ideótipos), criados com base nos dados experimentais para representar os genótipos de máxima adaptabilidade geral, máxima adaptabilidade específica a ambientes favoráveis ou desfavoráveis e os genótipos de mínima adaptabilidade. O ideótipo de máxima adaptabilidade geral é aquele que apresenta os valores máximos observados para todos os ambientes estudados (ideótipo I). Os ideótipos de máxima adaptabilidade específica são aqueles que apresentam máxima resposta em ambientes favoráveis e mínima resposta em ambientes desfavoráveis (ideótipo II) ou máxima resposta em ambientes desfavoráveis e mínima em ambientes favoráveis (ideótipo III). O ideótipo de mínima adaptabilidade é aquele que apresenta os menores valores em todos os ambientes estudados (ideótipo IV). Para utilização desse método, os ambientes foram classificados em favoráveis e desfavoráveis utilizando o índice ambiental como proposto por FINLAY e WILKINSON (1963).

$$
I_{j}=\frac{1}{g} \sum_{i} Y_{i j}-\frac{1}{a g} Y . .
$$

Em que: Yij: média do genótipo i, no ambiente j; $Y$.. : total das observações; a: número de ambientes; g: número de genótipos.

Após a classificação dos ambientes, foram criados pontos referenciais, os ideótipos de resposta diferenciada a ambientes favoráveis e desfavoráveis, visando a classificação dos outros pontos do gráfico considerando os valores de distância cartesiana entre os pontos a cada um dos quatro ideótipos. Uma medida de probabilidade espacial pode ser calculada utilizando o inverso da distância entre um tratamento aos quatro ideótipos:

$$
P_{d(i, j)}=\frac{\left[\frac{1}{d i}\right]}{\sum_{i=1}^{4} \frac{1}{d i}}
$$

Em que: $P_{d(i, j)}=$ probabilidade de apresentar padrão de estabilidade semelhante ao j-ésimo centróide; $d i=$ distância do i-ésimo ponto ao j-ésimo centróide.

\section{RESULTADOS E DISCUSSÃO}

Os coeficientes de variação experimental variaram de 10,4 a 15,5\% (Tabela 2), indicando certo controle das causas de variação de ordem sistemática dos ambientes experimentais, para a produtividade de grãos, que é um caráter quantitativo muito influenciado pelo ambiente. Segundo CARVALHO et al. (2003) 16\% é o limite máximo de coeficiente de variação para produtividade de grãos em soja.

Os efeitos da interação $\mathrm{G} \times \mathrm{A}$ apresentaram significância $(P<0,01)$ pelo teste $F$ (Tabela 3$)$. Os pares de ambientes Rondonópolis II - Nova Brasilândia e Nova Brasilândia - Vera apresentaram interação predominantemente do tipo simples, ou seja, parte complexa da interação menor que $50 \%$ (Tabela 4). Todos os outros pares de ambientes apresentaram interação do tipo complexa, ou seja, houve inconsistência na superioridade do cultivar com a variação ambiental, o que dificulta a indicação das cultivares e linhagens (CRUZ e CASTOLDI, 1991; VENCOVSKY e BARRIGA, 1992), pois não se pode, nessas circunstancias, fazer uma recomendação uniforme para todos os locais, sem prejuízo considerável na produção obtida, relativamente à produção possível. 
BARROS, H.B. et al. Análises paramétricas e não-paramétricas...

TABELA 3 - Análise de variância conjunta da produtividade de grãos $\left(\mathrm{kg} \mathrm{ha}^{-1}\right)$, de 30 genótipos de soja (ciclo precoce) avaliados em seis ambientes, no Estado do Mato Grosso, na safra 2004/2005

\begin{tabular}{|c|c|c|c|c|}
\hline Fonte de variação & $\mathrm{GL}$ & Quadrado médio & $\mathrm{F}$ & $\mathrm{Pr}>\mathrm{F}$ \\
\hline Blocos/ambientes & 18 & 220546,8708 & - & - \\
\hline Ambientes (A) & 5 & 9494296,9913 & 43,0488 & $<0,000$ \\
\hline Genótipos (G) & 29 & 1643262,7692 & 4,1162 & $<0,000$ \\
\hline Interação GxA & 145 & 399214,6339 & 2,7178 & $<0,000$ \\
\hline Erro médio & 522 & 146889,6648 & - & - \\
\hline
\end{tabular}

TABELA 4 - Pares de ambientes, correlação entre ambientes e porcentagem da parte complexa resultante da decomposição da interação entre genótipos e pares de ambiente, segundo metodologia de CRUZ e CASTOLDI (1991), nos ensaios de competição de linhagens de soja, de ciclo precoce, no Estado do Mato Grosso, na safra 2004/2005

\begin{tabular}{lcc}
\hline \multicolumn{1}{c}{ Pares de ambientes } & Correlação & Parte complexa da interação \\
\hline Rondonópolis I e Rondonópolis II & $-0,036$ & 99,986 \\
Rondonópolis I e Rondonópolis III & 0,137 & 73,256 \\
Rondonópolis I e Nova Brasilândia & 0,144 & 57,185 \\
Rondonópolis I e Campo Verde & 0,032 & 88,816 \\
Rondonópolis I e Vera & $-0,066$ & 92,093 \\
Rondonópolis II e Rondonópolis III & $0,436^{\star}$ & 62,409 \\
Rondonópolis II e Nova Brasilândia & $0,561^{\star *}$ & 37,662 \\
Rondonópolis II e Campo Verde & $0,453^{*}$ & 69,649 \\
Rondonópolis II e Vera & $0,576^{* *}$ & 58,386 \\
Rondonópolis III e Nova Brasilândia & 0,253 & 80,849 \\
Rondonópolis III e Campo Verde & $0,496^{\star *}$ & 67,857 \\
Rondonópolis III e Vera & $0,383^{*}$ & 76,845 \\
Nova Brasilândia e Campo Verde & $0,493^{* *}$ & 55,218 \\
Nova Brasilândia e Vera & $0,661^{* *}$ & 43,024 \\
Campo Verde e Vera & $0,556^{* *}$ & 66,446 \\
\hline
\end{tabular}

**, *: Significativo a 1 e $5 \%$ de probabilidade, respectivamente, pelo teste t.

O rendimento médio de grãos variou de $2837 \mathrm{~kg} \mathrm{ha}^{-1}$ (Rondonópolis III) a $3701 \mathrm{~kg} \mathrm{ha}^{-1}$ (Vera), com média geral entre os ambientes de $3200 \mathrm{~kg}$ ha $^{-1}$ (Tabela 5). A maior produtividade observada foi obtida pela linhagem SL 2238 (4336 $\left.\mathrm{kg} \mathrm{ha}^{-1}\right) \mathrm{com}$ média em todos os ambientes de $3725 \mathrm{~kg} \mathrm{ha}^{-1}$. A menor produtividade isolada foi obtida pela linhagem SL 1283 (1740 kg ha-1), entretanto, a menor produtividade em todos os ambientes foi obtida pela linhagem SL 1232 (2533 $\left.\mathrm{kg} \mathrm{ha}^{-1}\right)$.

As estimativas das médias dos genótipos, e parâmetros de adaptabilidade e estabilidade dos cultivares e linhagens obtidas pelos métodos de EBERHART e RUSSELL (1966), LIN e BINNS (1988) modificado por CARNEIRO (1998), ANNICCHIARICO (1992) e Centróide (ROCHA et al. 2005), encontramse nas Tabelas 6 e 7.

As linhagens SL 712, SL 202, SL 801, SL 2182, SL 1613 e os padrões Xingu, M-Soy 8329 e EMGOPA 315 apresentaram produtividade média elevada (superior a média geral), coeficientes de regressão estatisticamente igual a 1 e desvio da regressão não significativos. Portanto, pela metodologia de EBERHART e RUSSELL (1966) foram classificados como sendo de ampla adaptabilidade e alta estabilidade ou previsibilidade. Entretanto, com base nos coeficientes de determinação (R2), apenas a linhagem SL 801 apresentou ajustamento ideal à reta de regressão, o que indica alta confiabilidade na classificação desta linhagem como de alta previsibilidade de comportamento.

As linhagens SL 2238, SL 1949, SL 31, SL 2382 e os padrões Uirapuru e M-Soy 8411 apresentaram as maiores médias (acima de 3400 $\mathrm{kg}$ ha-1). Esses genótipos, por essa metodologia, possuem ampla adaptabilidade, pois apresentam coeficientes de regressão estatisticamente igual a $1\left(\beta_{1}=1\right)$. Entretanto, apresentam desvios da regressão significativos, ou seja, são de baixa estabilidade.

A linhagem SL 2435 apresentou elevada produtividade e coeficiente de regressão significativamente $<1$, sendo classificada como de adaptabilidade específica a ambientes desfavoráveis. Entretanto, apresentou baixa estabilidade, visto que o desvio da regressão foi significativo e o coeficiente de determinação mostrou-se de baixa magnitude $(R 2=2,48)$. 
BARROS, H.B. et al. Análises paramétricas e não-paramétricas...

TABELA 5 - Médias de produtividade de grãos de genótipos de soja de ciclo precoce em seis ambientes do estado do Mato Grosso, na safra 2004/2005 *

\begin{tabular}{|c|c|c|c|c|c|c|c|}
\hline \multirow{2}{*}{ Genótipos } & \multicolumn{6}{|c|}{ Ambientes } & \multirow{2}{*}{ Média } \\
\hline & RON. I & RON. II & RON. III & N. BRAS. & C. VERDE & VERA & \\
\hline SL 2238 & 2881 & 3594 & 3686 & 3878 & 3974 & 4336 & 3725 \\
\hline UIRAPURU & 3145 & 3251 & 3351 & 3872 & 3783 & 4151 & 3592 \\
\hline SL 1949 & 2893 & 3581 & 3076 & 4216 & 3378 & 4200 & 3557 \\
\hline SL 712 & 3463 & 3201 & 3294 & 3420 & 3829 & 4071 & 3546 \\
\hline XINGÚ & 3374 & 3370 & 3167 & 3477 & 3054 & 4022 & 3411 \\
\hline SL 31 & 2489 & 3545 & 3121 & 3535 & 3501 & 4185 & 3396 \\
\hline SL 2435 & 3464 & 3049 & 3060 & 4153 & 3135 & 3434 & 3382 \\
\hline M-SOY 8329 & 3479 & 3181 & 3202 & 2996 & 3678 & 3702 & 3373 \\
\hline EMGOPA 315 & 3069 & 3278 & 3286 & 3290 & 3285 & 3726 & 3322 \\
\hline M-SOY 8411 & 3274 & 3431 & 3408 & 2816 & 2910 & 3811 & 3275 \\
\hline SL 202 & 3314 & 3340 & 2978 & 3204 & 2894 & 3910 & 3273 \\
\hline SL 801 & 3299 & 3073 & 2939 & 2982 & 3389 & 3883 & 3261 \\
\hline SL 2382 & 3241 & 3245 & 2346 & 3765 & 3247 & 3691 & 3256 \\
\hline SL 2182 & 3071 & 3415 & 2652 & 3563 & 3126 & 3620 & 3241 \\
\hline SL 1613 & 3133 & 3463 & 2544 & 3348 & 3287 & 3665 & 3240 \\
\hline SL 9 & 3011 & 3026 & 2900 & 3197 & 3319 & 3735 & 3198 \\
\hline SL 1769 & 3218 & 3300 & 2292 & 3593 & 2904 & 3881 & 3198 \\
\hline SL 36 & 3089 & 3412 & 2326 & 3111 & 3364 & 3853 & 3192 \\
\hline SL 1132 & 3016 & 2816 & 2783 & 3161 & 3337 & 3953 & 3177 \\
\hline SL 13 & 3071 & 3109 & 2438 & 3219 & 3210 & 3961 & 3168 \\
\hline CONQUISTA & 3131 & 3368 & 2984 & 2867 & 3062 & 3246 & 3110 \\
\hline SL 120 & 2952 & 2962 & 2583 & 3466 & 3126 & 3450 & 3090 \\
\hline SL 11 & 3177 & 2996 & 3278 & 2450 & 3168 & 3413 & 3080 \\
\hline SL 12 & 2983 & 3066 & 1875 & 3096 & 3362 & 3845 & 3038 \\
\hline SL 1148 & 3227 & 2937 & 2618 & 2586 & 3319 & 3345 & 3005 \\
\hline SL 1270 & 3018 & 3109 & 2825 & 2572 & 3535 & 2918 & 2996 \\
\hline SL 1136 & 3082 & 2889 & 2872 & 2335 & 3068 & 3433 & 2946 \\
\hline SL 1283 & 2782 & 3211 & 2794 & 1740 & 2860 & 3308 & 2782 \\
\hline SL 1264 & 2748 & 2515 & 2379 & 2220 & 2593 & 3453 & 2651 \\
\hline SL 1232 & 3179 & 2588 & 2043 & 2235 & 2335 & 2818 & 2533 \\
\hline Média & 3109 & 3177 & 2837 & 3145 & 3234 & 3701 & 3200 \\
\hline
\end{tabular}

* DMS Tukey a 5\% de probabilidade entre ambientes $=772,27 \mathrm{~kg} \mathrm{ha}^{-1}$ e entre genótipos $=1015,64 \mathrm{~kg} \mathrm{ha}^{-1}$. CV $(\%)=11,97$.

A linhagem SL 2238 apresentou a maior média de produtividade e menores valores de $P_{i}$ geral, favorável e desfavorável, seguida da cultivar padrão Uirapuru, sendo, portanto, indicadas pela metodologia de LIN e BINNS (1988) como de alta estabilidade. Entretanto, pela metodologia de EBERHART e RUSSELL (1966) tal genótipo foi classificado como de baixa estabilidade. A linhagem SL 712 apresentou baixo valor de $P_{i}$, sendo classificada como de alta estabilidade, coincidindo com a classificação obtida pela metodologia de EBERHART e RUSSELL (1966). Entretanto, as linhagens SL 202, SL 801, SL 2182, SL 1613 e as cultivares padrão Xingu e Emgopa 315, classificadas como estáveis pelo método de EBERHART e RUSSELL (1966), apresentaram $P_{\text {is }}$ intermediários pelo método de LIN e BINNS (1988), sendo, portanto, classificadas como de média estabilidade. A linhagem SL 31 mostrou-se mais adaptada a ambientes favoráveis, diferindo da classificação da linhagem SL 2435 que mostrou ser mais adaptada a ambientes desfavoráveis.

Com base no índice de confiança ou de recomendação $\left(W_{i}\right)$, proposto por ANNICCHIARICO (1992), pode-se indicar os genótipos SL 2238, Uirapuru, SL 712, SL 1949 e Xingu como os cinco de melhor adaptação, considerando os seus comportamentos em todos os ambientes. Nos ambientes favoráveis (Campo Verde e Vera) os genótipos SL 2238, Uirapuru, SL 712, SL 31 e SL 1949 , foram os cinco com comportamentos mais estáveis. Nos ambientes classificados como 
BARROS, H.B. et al. Análises paramétricas e não-paramétricas...

TABELA 6 - Produtividade média $\left(\mathrm{kg} \mathrm{ha}^{-1}\right)$, estimativas dos coeficientes de regressão $\left(\hat{\beta}_{\mathrm{i}}\right)$, dos desvios da regressão $\left(\mathcal{G}_{d i}^{2}\right)$, coeficiente de determinação $\left(\mathrm{R}^{2}\right), P_{i s}$ (geral, favorável e desfavorável), dos genótipos de soja de ciclo precoce, no estado do Mato Grosso, na safra 2004/2005

\begin{tabular}{|c|c|c|c|c|c|c|c|}
\hline \multirow[b]{2}{*}{ Genótipos } & \multirow[b]{2}{*}{ Média } & \multicolumn{3}{|c|}{ Eberhart e Russell (1966) } & \multicolumn{3}{|c|}{ Lin e Binns (1988) } \\
\hline & & $\hat{\beta}_{1 i}$ & $\mathcal{\sigma}_{d i}^{2}$ & $\mathrm{R}^{2}$ & Geral & Fav. & Desfav. \\
\hline SL 2238 & 3725 & $1,009^{\text {ns }}$ & 159687,8 & 33,93 & 611,1 & 271,0 & 340,1 \\
\hline UIRAPURU & 3592 & $1,029^{\text {ns }}$ & $58964,2^{*}$ & 52,26 & 724,4 & 358,5 & 365,9 \\
\hline SL 1949 & 3557 & $1,300^{\text {ns }}$ & $184240,4^{\star \star}$ & 43,09 & 868,9 & 441,4 & 427,4 \\
\hline SL 712 & 3546 & $0,976^{\mathrm{ns}}$ & $9248,5^{\mathrm{ns}}$ & 67,23 & 707,5 & 366,5 & 341,0 \\
\hline XINGÚ & 3411 & $0,967^{\mathrm{ns}}$ & $12487,9^{\text {ns }}$ & 65,31 & 943,2 & 558,6 & 384,6 \\
\hline SL 31 & 3396 & $1,440^{\mathrm{ns}}$ & $151175,8^{* *}$ & 52,19 & 921,3 & 416,4 & 504,9 \\
\hline SL 2435 & 3382 & $0,234^{+}$ & $177511,8^{\star *}$ & 2,48 & 1050,8 & 676,6 & 374,2 \\
\hline M-SOY 8329 & 3373 & $0,638^{\mathrm{ns}}$ & $28226,1^{\text {ns }}$ & 38,28 & 858,3 & 487,7 & 370,6 \\
\hline EMGOPA 315 & 3322 & $0,606^{\text {ns }}$ & $-14838,9^{\text {ns }}$ & 62,41 & 993,3 & 573,9 & 419,4 \\
\hline M-SOY 8411 & 3275 & $0,579^{\text {ns }}$ & $98479,7^{* *}$ & 19,69 & 1032,5 & 641,4 & 391,1 \\
\hline SL 202 & 3273 & $1,040^{\text {ns }}$ & $18164,2^{\text {ns }}$ & 66,12 & 1056,8 & 622,1 & 434,8 \\
\hline SL 801 & 3261 & $1,134^{\mathrm{ns}}$ & $-8543,3^{\text {ns }}$ & 81,86 & 960,6 & 512,9 & 447,7 \\
\hline SL 2382 & 3256 & $1,319^{\text {ns }}$ & $110220,2^{* *}$ & 53,94 & 1098,0 & 590,8 & 507,2 \\
\hline SL 2182 & 3241 & $0,995^{\mathrm{ns}}$ & $32032,6^{\text {ns }}$ & 58,78 & 1130,0 & 635,5 & 494,5 \\
\hline SL 1613 & 3240 & $1,178^{\mathrm{ns}}$ & $11063,5^{\mathrm{ns}}$ & 74,19 & 1087,8 & 587,5 & 500,3 \\
\hline SL 1769 & 3198 & $1,605^{+}$ & $93462,2^{* *}$ & 66,21 & 1058,0 & 563,6 & 494,4 \\
\hline SL 9 & 3198 & $1,017^{\text {ns }}$ & $-24873,4^{\text {ns }}$ & 89,63 & 1157,7 & 626,4 & 531,3 \\
\hline SL 36 & 3192 & $1,669^{+}$ & $8249,1^{\text {ns }}$ & 85,98 & 1070,6 & 525,5 & 545,0 \\
\hline SL 1132 & 3177 & $1,417^{\text {ns }}$ & $-615,1^{\text {ns }}$ & 84,63 & 1028,4 & 508,8 & 519,6 \\
\hline SL 13 & 3168 & $1,695^{+}$ & $-26590,0^{\text {ns }}$ & 96,56 & 1083,1 & 536,6 & 546,6 \\
\hline CONQUISTA & 3110 & $0,297^{+}$ & $-4713,7^{\mathrm{ns}}$ & 21,44 & 1205,3 & 737,6 & 467,6 \\
\hline SL 120 & 3090 & $0,911^{\text {ns }}$ & $22292,7^{\text {ns }}$ & 58,22 & 1220,9 & 675,2 & 545,8 \\
\hline SL 11 & 3080 & $0,316^{+}$ & $96215,7^{\star *}$ & 6,93 & 1122,2 & 673,9 & 448,2 \\
\hline SL 12 & 3038 & $2,120^{++}$ & $47622,2^{\text {ns }}$ & 84,06 & 1167,0 & 528,2 & 638,8 \\
\hline SL 1148 & 3005 & $0,823^{\text {ns }}$ & $44601,5^{\text {ns }}$ & 45,20 & 1178,9 & 654,7 & 524,2 \\
\hline SL 1270 & 2996 & $0,152^{++}$ & $90624,1^{* *}$ & 1,78 & 1218,9 & 703,9 & 515,0 \\
\hline SL 1136 & 2946 & $0,751^{\text {ns }}$ & $70544,1^{*}$ & 34,25 & 1222,6 & 692,7 & 529,9 \\
\hline SL 1283 & 2782 & $0,781^{\mathrm{ns}}$ & $291098,8^{* *}$ & 15,55 & 1369,1 & 770,3 & 598,7 \\
\hline SL 1264 & 2651 & $1,304^{\mathrm{ns}}$ & $28505,5^{\text {ns }}$ & 72,08 & 1481,3 & 798,6 & 682,7 \\
\hline SL 1232 & 2533 & $0,683^{\text {ns }}$ & $134559,1^{* \star}$ & 21,23 & 1672,2 & 1006,8 & 665,4 \\
\hline
\end{tabular}

desfavoráveis (Rondonópolis I, II, III e Nova Brasilândia) os cinco genótipos mais estáveis foram: SL 2238, Xingu, Uirapuru, SL 2435 e SL 712. Comparando a classificação da estabilidade fenotípica dos genótipos obtida pela metodologia proposta por ANNICCHIARICO (1992), com classificação obtida pela metodologia de LIN e BINNS (1988), observou-se que os cinco melhores genótipos indicados pela metodologia de Annicchiarico como sendo de estabilidade geral coincidem com a classificação obtida pela metodologia de LIN e BINNS (1988), com exceção da cultivar M-Soy 8329, que foi classificada como a $4^{\text {a }}$ e 6a mais estável pelas metodologias de LIN e BINNS (1988) e ANNICCHIARICO (1992), respectivamente. Nos ambientes classificados como favoráveis e desfavoráveis, houve coincidência entre os cinco melhores genótipos em ambas as metodologias, exceto para a cultivar Xingu, que apresentou o segundo maior índice de confiança para ambientes favoráveis, pela metodologia de ANNICCHIARICO (1992) e o sexto menor $P_{i}$ pela metodologia de LIN e BINNS (1988).

O conceito de adaptabilidade e estabilidade utilizado no método centróide diferencia dos demais, uma vez que o genótipo de máxima adaptação específica não é aquele que apresenta bom desempenho nos grupos de ambientes favoráveis ou desfavoráveis, mas sim o genótipo que apresenta valores máximos para determinado grupo de ambientes (favoráveis e desfavoráveis) e mínimo para o outro conjunto (ROCHA et al., 2005). 
TABELA 7 - Produtividade média $\left(\mathrm{kg} \mathrm{ha}^{-1}\right), W_{\text {is }}$ (geral, favorável e desfavorável) e a classificação dos genótipos em um dos quatro grupos caracterizados pelos centróides e a probabilidade associada a sua classificação dos genótipos de soja de ciclo precoce, no estado do Mato Grosso, na safra 2004/ 2005

\begin{tabular}{|c|c|c|c|c|c|c|c|c|c|}
\hline \multirow{2}{*}{ Genótipos } & \multirow{2}{*}{ Média } & \multicolumn{3}{|c|}{ Annicchiarico (1992) } & \multicolumn{5}{|c|}{ Centróide $^{2 t}$} \\
\hline & & Geral & Fav. & Desfav. & Grupo & $\mathrm{P}(\mathrm{I})$ & $\mathrm{P}(\mathrm{II})$ & $P(I I I)$ & $\mathrm{P}(\mathrm{IV})$ \\
\hline SL 2238 & 3725 & 112,9 & 110,3 & 118,9 & 1 & 0,587 & 0,133 & 0,173 & 0,107 \\
\hline UIRAPURU & 3592 & 109,9 & 108,1 & 113,6 & I & 0,548 & 0,143 & 0,192 & 0,117 \\
\hline SL 1949 & 3557 & 107,3 & 107,4 & 107,2 & I & 0,457 & 0,157 & 0,248 & 0,139 \\
\hline SL 712 & 3546 & 109,2 & 107,5 & 112,6 & I & 0,456 & 0,184 & 0,213 & 0,146 \\
\hline XINGÚ & 3411 & 104,9 & 108,5 & 98,8 & I & 0,360 & 0,182 & 0,288 & 0,170 \\
\hline SL 31 & 3396 & 102,4 & 99,2 & 109,7 & 1 & 0,373 & 0,215 & 0,237 & 0,176 \\
\hline SL 2435 & 3382 & 102,2 & 107,7 & 94,1 & III & 0,324 & 0,152 & 0,368 & 0,156 \\
\hline M-SOY 8329 & 3373 & 103,4 & 102,6 & 104,2 & 1 & 0,336 & 0,227 & 0,245 & 0,192 \\
\hline EMGOPA 315 & 3322 & 102,4 & 103,6 & 100,9 & I & 0,336 & 0,197 & 0,282 & 0,185 \\
\hline M-SOY 8411 & 3275 & 99,5 & 102,3 & 94,0 & III & 0,285 & 0,212 & 0,289 & 0,213 \\
\hline SL 202 & 3273 & 100,5 & 104,1 & 94,4 & I & 0,297 & 0,208 & 0,290 & 0,205 \\
\hline SL 801 & 3261 & 100,5 & 98,8 & 104,8 & I & 0,300 & 0,251 & 0,238 & 0,211 \\
\hline SL 2382 & 3256 & 98,3 & 98,0 & 99,9 & I & 0,305 & 0,214 & 0,278 & 0,204 \\
\hline SL 2182 & 3241 & 99,2 & 100,8 & 97,0 & I & 0,298 & 0,203 & 0,296 & 0,202 \\
\hline SL 1613 & 3240 & 99,3 & 99,1 & 99,8 & I & 0,295 & 0,227 & 0,266 & 0,213 \\
\hline SL 1769 & 3198 & 96,2 & 96,7 & 94,4 & I & 0,280 & 0,222 & 0,277 & 0,221 \\
\hline SL 9 & 3198 & 99,1 & 98,0 & 101,5 & I & 0,293 & 0,239 & 0,253 & 0,216 \\
\hline SL 36 & 3192 & 96,8 & 94,0 & 104,1 & II & 0,271 & 0,271 & 0,229 & 0,229 \\
\hline SL 1132 & 3177 & 97,3 & 94,6 & 104,3 & I & 0,284 & 0,266 & 0,230 & 0,220 \\
\hline SL 13 & 3168 & 96,6 & 94,3 & 101,6 & I & 0,273 & 0,265 & 0,234 & 0,228 \\
\hline CONQUISTA & 3110 & 95,5 & 98,9 & 89,8 & III & 0,244 & 0,218 & 0,290 & 0,248 \\
\hline SL 120 & 3090 & 94,7 & 95,0 & 94,3 & III & 0,262 & 0,224 & 0,279 & 0,235 \\
\hline SL 11 & 3080 & 93,3 & 93,2 & 94,0 & IV & 0,237 & 0,251 & 0,248 & 0,264 \\
\hline SL 12 & 3038 & 90,3 & 85,0 & 103,9 & II & 0,228 & 0,313 & 0,203 & 0,256 \\
\hline SL 1148 & 3005 & 91,7 & 90,3 & 94,1 & IV & 0,216 & 0,279 & 0,219 & 0,286 \\
\hline SL 1270 & 2996 & 90,9 & 91,8 & 88,2 & IV & 0,221 & 0,259 & 0,236 & 0,284 \\
\hline SL 1136 & 2946 & 89,6 & 88,0 & 93,4 & IV & 0,202 & 0,275 & 0,214 & 0,309 \\
\hline SL 1283 & 2782 & 82,5 & 80,3 & 88,7 & IV & 0,167 & 0,272 & 0,184 & 0,377 \\
\hline SL 1264 & 2651 & 80,4 & 78,4 & 84,2 & IV & 0,142 & 0,251 & 0,161 & 0,446 \\
\hline SL 1232 & 2533 & 75,9 & 77,7 & 73,4 & IV & 0,127 & 0,189 & 0,163 & 0,521 \\
\hline
\end{tabular}

"Alfa = 0,25; Z(1-alfa) = 0,2734. ${ }^{2 /}$ Em que: Ideótipo I = Adaptabilidade geral (++); Ideótipo II = Adaptabilidade especifica a ambientes favoráveis (+-); Ideótipo III = Adaptabilidade específica a ambientes desfavoráveis (-+); Ideótipo IV = Pouco adaptado (--).

Após a classificação dos ambientes e a estimação dos os ideótipos (Tabela 8), pelo método centróide (ROCHA et al., 2005) estimados com base nos dados originais foram acrescidos na análise (Tabela 8), utilizou-se a análise de componentes principais envolvendo os 30 genótipos iniciais e quatro outros representativos, que na analise gráfica, representam os quatro centróides em torno dos quais foi avaliada a dispersão dos demais. A obtenção dos autovalores, via metodologia dos componentes principais, partindo dos dados originais incluídos os ideótipos, mostra que apenas dois componentes principais são suficientes para explicar proporções superiores a $74 \%$ da variação total (Tabela 9). Uma vez constatada a suficiência de dois autovalores na representação da variação total, a avaliação da posição dos genótipos pode ser feita por meio de gráficos bidimensionais (CARVALHO et al., 2002).

A análise visual do gráfico da análise de componentes principais permitiu avaliar que, os genótipos apresentaram distribuição heterogênea para a produtividade de grãos e que existem pontos de maior proximidade a todos os quatro centróides, possibilitando uma recomendação de genótipos de adaptabilidade geral ou recomendação de genótipos de adaptabilidade específica a um subgrupo de ambientes. Os seguintes genótipos: 22 (SL 2238), 29 (Uirapuru), 20 (SL 1949) e 9 (SL 712) foram classificados como sendo de adaptabilidade geral, por localizarem-se mais próximos do ideótipo I. Entretanto, a maioria dos pontos (genótipos) foram plotados na região central do gráfico, dificultando a classificação. Neste caso, utilizou-se o inverso do valor da distância entre um ponto aos quatro centróides como estimativa da confiabilidade de agrupamento dos genótipos (ROCHA et al., 2005). Dessa maneira, um ponto eqüidistante aos quatro pontos referenciais apresenta valores de probabilidade de $25 \%$ de pertencer a qualquer um dos grupos e, portanto, quanto mais o valor de probabilidade diferir de $25 \%$, maior será a certeza em concluir o agrupamento do genótipo. Segundo ROCHA et al. (2005), valores de probabilidade próximos ou superiores a $50 \%$ indicam boa confiabilidade no agrupamento. 
BARROS, H.B. et al. Análises paramétricas e não-paramétricas...

TABELA 8 - Classificação dos ambientes utilizando o índice ambiental e estabelecimento dos ideótipos, calculados pelo método CENTRÓIDE, dos genótipos de soja de ciclo precoce, no estado do Mato Grosso, na safra 2004/2005

\begin{tabular}{|c|c|c|c|c|c|c|c|c|}
\hline \multirow{2}{*}{ Ambientes } & \multirow{2}{*}{$\begin{array}{c}\text { Média } \\
\left(\mathrm{kg} \mathrm{ha}^{-1}\right)\end{array}$} & \multirow[b]{2}{*}{$\mathrm{l}_{\mathrm{j}}$} & \multicolumn{2}{|c|}{ Produtividade } & \multicolumn{4}{|c|}{ Ideótipos } \\
\hline & & & Máxima & Mínima & I & II & III & IV \\
\hline Rondonópolis I & 3109 & $-91,43$ & 3479 & 2489 & 3479 & 2489 & 3479 & 2489 \\
\hline Rondonópolis II & 3177 & $-23,19$ & 3594 & 2515 & 3594 & 2515 & 3594 & 2515 \\
\hline Rondonópolis III & 2837 & $-363,97$ & 3686 & 1875 & 3686 & 1875 & 3686 & 1875 \\
\hline N. Brasilândia & 3145 & $-55,20$ & 4216 & 1740 & 4216 & 1740 & 4216 & 1740 \\
\hline Campo Verde & 3234 & 33,76 & 3974 & 2335 & 3974 & 3974 & 2335 & 2335 \\
\hline Vera & 3701 & 500,03 & 4336 & 2818 & 4336 & 4336 & 2818 & 2818 \\
\hline
\end{tabular}

Em que: Ideótipo I = Adaptabilidade geral (++); Ideótipo II = Adaptabilidade especifica a ambientes favoráveis (+-); Ideótipo III = Adaptabilidade específica a ambientes desfavoráveis (-+); Ideótipo IV = Pouco adaptado (--).

TABELA 9 - Estimativa dos autovalores obtidos conforme a técnica de componentes principais e fração cumulativa da variância explicada por estes a partir da avaliação de genótipos de soja de ciclo precoce, no estado do Mato Grosso, na safra 2004/2005

\begin{tabular}{ccc}
\hline Raiz & Raiz $(\%)$ & \% Acumulada \\
\hline 3,03095 & 50,52 & 50,51 \\
1,45619 & 24,27 & 74,78 \\
0,57920 & 9,65 & 84,44 \\
0,49314 & 8,22 & 92,66 \\
0,22508 & 3,75 & 96,41 \\
0,21541 & 3,59 & 100,0 \\
\hline
\end{tabular}

As classificações dos genótipos a um dos quatro grupos e a probabilidade associada a sua classificação são apresentadas na Tabela 7. Pelo método Centróide os genótipos: SL 2238, Uirapuru, SL 1949, SL 712, Xingu, SL 31, M-Soy 8329, EMGOPA 315, SL 202, SL 801, SL 2382, SL 2182, SL 1613, SL 9, SL 1769, SL 1132 e SL 13 foram classificados para amplas condições ambientais (grupo I) com destaque para os genótipos SL 2238, Uirapuru, SL 1949, SL 712 e Xingu, alem da linhagem SL 2435 que foi classificada como de adaptabilidade específica a ambientes desfavoráveis.

Observa-se uma tendência de aumento na média de produtividade dos genótipos de soja à medida que estes se aproximam do centróide I (adaptabilidade geral), conforme Figura 1 e Tabela 7. De acordo com ROCHA et al. (2005), quanto menor for a diferença entre um genótipo qualquer e o ideótipo I, menor será a diferença entre este e o genótipo de máximo desempenho em todos os ambientes, fazendo com que a adaptabilidade geral esteja necessariamente associada ao melhor desempenho.

Comparando a classificação da estabilidade fenotípica dos genótipos obtida pelo método Centróide com classificação obtida pela metodologia de ANNICCHIARICO (1992), observase que os genótipos SL 2238, Uirapuru, SL 1949,
SL 712 e Xingu foram classificados como sendo de adaptabilidade geral.

\section{CONCLUSÕES}

Pela análise de regressão proposta por EBERHART e RUSSELL, os genótipos mais produtivos SL 2238, UIRAPURU e SL 1949, apresentam baixa previsibilidade;

As metodologias de LIN e BINNS, ANNICCHIARICO e Centróide são coerentes entre si e permitem identificar, entre os genótipos avaliados, os de maior produtividade, estabilidade e adaptabilidade;

Com base nas metodologias de LIN e BINNS, ANNICCHIARICO e Centróide as linhagens de soja SL 2238, SL 1949 e SL 712 apresentam superioridade em termos de adaptabilidade, estabilidade e performance para a produtividade de grãos.

\section{AGRADECIMENTOS}

Ao Conselho Nacional de Desenvolvimento Científico e Tecnológico (CNPq) pelo apoio através da concessão de bolsa em nível de Doutorado e as empresas Bacuri/Soygene e Sales Agropecuária pela condução dos ensaios de campo. 
BARROS, H.B. et al. Análises paramétricas e não-paramétricas...

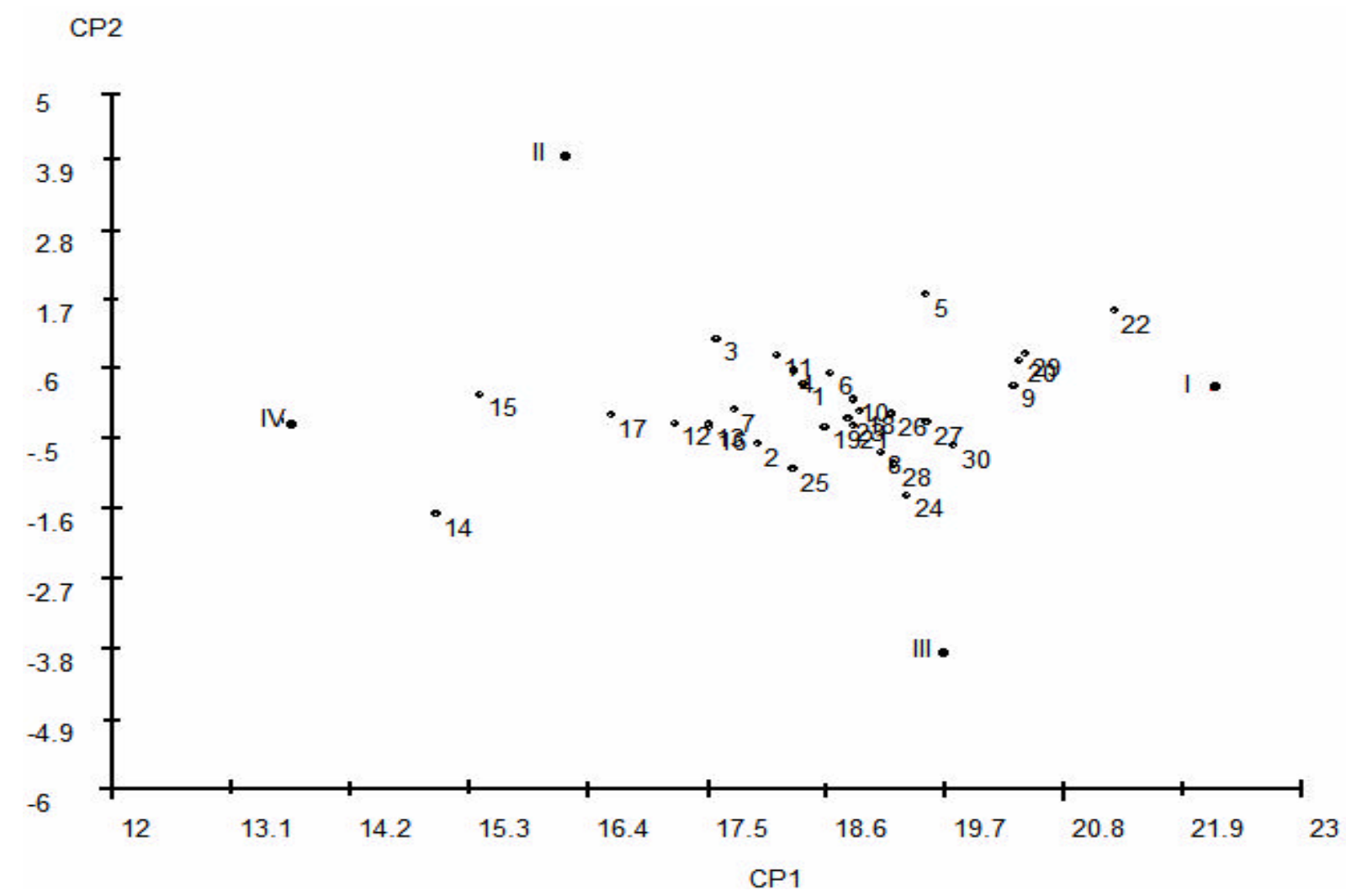

FIGURA 1 - Dispersão gráfica dos escores em relação aos dois primeiros componentes principais obtidos da análise da produtividade de 30 genótipos de soja avaliados em seis ambientes, Os quatro pontos numerados com algarismos romanos representam os centróides. $\mathbf{1}=\mathrm{SL}$ 9; 2 = SL 11; 3 = SL 12; 4 = SL 13; 5 = SL 31; $\mathbf{6}=$ SL 36; 7 = SL 120; 8 = SL 202; 9 = SL 712; 10 = SL 801; 11 = SL $1132 ; 12$ = SL 1136; 13 = SL 1148; 14 = SL 1232; 15 = SL 1264; 16 = SL 1270; 17 = SL 1283; 18 = SL 1613; $19=$ SL 1769; 20 = SL 1949; 21 = SL 2182; 22 = SL 2238; 23 = SL 2382; 24 = SL 2435; 25 = CONQUISTA; 26 = EMGOPA 315; 27 = M-SOY 8329; 28 = M-SOY 8411; 29 = UIRAPURU e 30 = XINGÚ.

\section{REFERÊNCIAS}

1. ANNICCHIARICO, P. Cultivar adaptation and recommendation from alfafa trials in Northern Italy. Journal of Genetics and Breeding, v. 46, n. 1, p. 269-278, 1992.

2. ATROCH, A. L.; SOARES, A. A.; RAMALHO, M. A. P. Adaptabilidade e estabilidade de linhagens de arroz de sequeiro testadas no Estado de Minas Gerais. Ciência e Agrotecnologia, v. 24, n. 3, p. 541-548, 2000.

3. CARNEIRO, P. C. S. Novas metodologias de análise de adaptabilidade e estabilidade de comportamento. $168 \mathrm{f}$. Tese (Doutorado em Genética e Melhoramento) - Universidade Federal de Viçosa, Viçosa, 1998.

4. CARVALHO, C. G. P.; ARIAS, C. A. A.; TOLEDO, J. F. F.; ALMEIDA, L. A.; KIIHL, R. A. S.; OLIVEIRA, M. F.; HIROMOTO, D. M.; TAKEDA, C. Proposta de classificação dos coeficientes de variação em relação à produtividade e altura da planta de soja. Pesquisa Agropecuária Brasileira, v. 38, n. 2, p. 187-193, 2003.

5. CARVALHO, H. W. L.; SILVA, M. L.; CARDOSO, M. J.; SANTOS, M. X.; TABOSA, J. N.; CARVALHO, C. L.; LIRA, M. A. Adaptabilidade e estabilidade de cultivares de milho no Nordeste brasileiro no triênio de 1998 a 2000. Pesquisa Agropecuária Brasileira, v. 37, n. 11, p. 1581-1588, 2002.

6. CRUZ, C. D. Programa GENES - aplicativo computacional em genética e estatística, 2001. $542 \mathrm{p}$.

7. CRUZ, C. D.; CASTOLDI, F. L. Decomposição da interação genótipos ambientes em partes simples e complexa. Revista Ceres, v. 38, n. 219, p. 422-430, 1991.

8. CRUZ, C. D.; REGAZZI, A. J. Modelos biométricos aplicados ao melhoramento genético. Viçosa: Editora UFV, 1997. $390 \mathrm{p}$.

9. CRUZ, C. D.; TORRES, R. A.; VENCOVSKY, R. An alternative approach to the stability analysis proposed by Silva and Barreto. Revista Brasileira de Genética, v. 12, p. 567-580, 1989.

10. EBERHART, S. A.; RUSSELL, W. A. Stability parameters for comparing varieties. Crop Science, v. 6, n. 1, p. 36-40, 1966. 
11. FARIAS, F. J. C.; RAMALHO, M. A. P. R.; CARVALHO, L. P.; MOREIRA, J. A. N.; COSTA, J. N. Parâmetros de estabilidade propostos por Lin \& Binns (1988) comparados com o método da regressão. Pesquisa Agropecuária Brasileira, v. 32, v. 4, p. 407-414, 1997.

12. FINLAY, K. W.; WILKINSON, G. N. The analysis of adaptation in plant-breeding programme. Australian Journal of Agricultural Research, v. 14, n. 5, p. 742-754, 1963.

13. FREEMAN, G. H.; PERKINS, J. M. Environmental and genotype-environmental components of variability. VIII. Relations between genotypes grown in different environments and measures of these environments. Heredity, v. 27, p. 15-23, 1971.

14. LIN, C. S.; BINNS, M. R. A superiority measure of cultivar performance for cultivars $x$ location data. Canadian Journal of Plant Science, v. 68, n. 1, p. 193-198, 1988.

15. LIN, C. S.; BINNS, M. R.; LEFKOVITCH, L. P. Stability analysis: Where do we stand? Crop Science, v. 26, p. 894-900, 1986

16. MAIA, M. C. C.; VELLO, N. A.; ROCHA, M. M.; PINHEIRO, J. B.; SILVA JUNIOR, N. F. Adaptabilidade e estabilidade de linhagens experimentais de soja selecionadas para caracteres agronômicos através de método uni-multivariado. Bragantia, v. 65, n. 2, p. 215-226, 2006.

17. NUNES, G. H. S.; REZENDE, G. D. S. P. M.; RAMALHO, M. A. P.; SANTOS, J. B. S.; Implicações da interação genótipos $x$ ambientes na seleção de clones de eucalipto. Cerne, v. 8, n. 1, p. 49-58, 2002.

18. PERKINS, J. M.; JINKS, J. L. Environmental and genotype-environmental components of variability. III. Multiple lines and crosses. Heredity, v. 23, p. 339-356, 1968.

19. PIMENTEL-GOMES, F. Curso de estatística experimental. 13. ed. Nobel. 1990. $468 \mathrm{p}$

20. PLAISTED, R. L.; PETERSON, L. C. A technique for evaluating the ability of selections to yield consistently in different locations or seasons. American Potato Journal, v. 36, p. 381-385, 1959.

21. PRADO, E. E. P.; HIRIMOTO, D. M.; GODINHO, V. P. C.; UTUMI, M. M. RAMALHO, A. R. Adaptabilidade e estabilidade de cultivares de soja em cinco épocas de plantio no cerrado de Rondônia. Pesquisa Agropecuária Brasileira, v. 36, n. 4, p. 625-635, 2001.

22. RAMALHO, M. P.; SANTOS, J. B.; ZIMMERMANN, M. J. Interação dos genótipos por ambientes. In: RAMALHO, M. P. SANTOS, J. B.; ZIMMERMANN, M. J. Genética quantitativa em plantas autógamas: aplicações ao melhoramento do feijoeiro. Gioiânia: Editora da UFG, 1993. p. 137-170.

23. ROCHA, R. B.; MURO-ABAD, J. I.; ARAUJO, E. F.; CRUZ, C. D. Avaliação do método centróide para estudo de adaptabilidade ao ambiente de clones de Eucalyptus grandis. Ciência Florestal, v. 15, n. 3, p. 255-266, 2005.

24. TAI, G. C. C. Genotype stability analysis and its a application to potato regional trials. Crop Science, v. 11, p. 184-190, 1971.

25. SILVA, J. G. C.; BARRETO, J. N. An application of segmented linear regression to the study of genotype $x$ environment interaction. Biometrics, v. 41, n. 4, p. 1093, 1986.

26. SILVA, W. C. J.; DUARTE, J. B. Métodos estatísticos para estudo de adaptabilidade e estabilidade fenotipica em soja. Pesquisa Agropecuária Brasileira, v. 41, n. 1, p. 23-30, 2006

27. VENCOVSKY, R.; BARRIGA, P. Genética biométrica no fitomelhoramento. Ribeirão Preto: Sociedade Brasileira de Genética, 1992. $486 \mathrm{p}$

28. WRICKE, G. Zur Berechnung der Ökovalenz bei Sommerweizen und Hafer. Zeitschrift für Pflanzenzüchtung, v. 52, p. 127-138, 1965. 

